\title{
Opportunities for Oral Health Monitoring Technologies Beyond the Dental Clinic
}

\author{
Adele Parsons \\ Human Centered Design and Engineering \\ University of Washington \\ Seattle Washington U.S. \\ adelepa@uw.edu
}

\author{
Chia-Fang Chung \\ Human Centered Design and Engineering \\ University of Washington \\ Seattle Washington U.S. \\ cfchung@uw.edu
}

\author{
Molly Donohue \\ Human Centered Design and Engineering \\ University of Washington \\ Seattle Washington U.S. \\ donoh95@uw.edu
}

\author{
Sean A. Munson \\ Human Centered Design and Engineering \\ University of Washington \\ Seattle Washington U.S. \\ smunson@uw.edu
}

Eric J. Seibel

Mechanical Engineering

University of Washington

Seattle Washington U.S.

eseibel@uw.edu

\begin{abstract}
Personal health and wellness technologies can improve people's care at home, connect everyday activities to clinical settings, and allow more efficient use of clinical resources. Recently, the Human-Computer Interaction community has begun to develop tools to improve oral care. In this research, we investigate dental practices and information needs through surveys and interviews with a range of patients and oral health providers. We find that personal users want to track their progress-or lack thereof-between dental visits for feedback, so they can adjust their home care routines, or so they can seek an escalation in care if they identify a problem. Among providers and clinical health workers, there exists an opportunity for better screening and diagnostic tools to identify dental caries at early stages. Providers in rural areas desire better tools to communicate problem areas to patients and their caregivers to bridge oral health care disparities in areas with limited access to care. Our results can guide the development of dental technologies that can address currently unmet needs of patients and providers.
\end{abstract}

\section{Author Keywords}

Health, Oral Health, Personal Informatics.

\section{ACM Classification Keywords}

H.5.m. Information interfaces and presentation (e.g., HCI): Miscellaneous

\section{ACM Reference format:}

PervasiveHealth '18, May 21-24, 2018, New York, NY, USA (C) 2018 Copyright is held by the owner/author(s). Publication rights licensed to ACM. ACM 978-1-4503-6450-8/18/05 \$15.00 https://doi.org/10.1145/3240925.3240973

\section{INTRODUCTION}

Lack of access to oral health care and inadequate knowledge of proper oral hygiene leads to an abundance of oral diseases in the United States. Education about proper oral hygiene techniques is important because dental caries is the single most common chronic disease of children aged 6 to 19 years old and occurs in some degree to 9 out of 10 adults over the age of 20 [1]. Cost is one of the main reasons that prevents adults from receiving regular dental care, along with proximity to dental providers [2]. Adults and children living in rural populations with increased vulnerability to oral health diseases, like Alaskan Native communities, experience significant disparities in oral health care [3]. In Alaska, Dental Health Aide Therapists (DHATs) work to bridge oral health disparities by providing preventative care and tooth extractions to children and adults [3]. While increasingly common, dental caries can be avoided with proper oral hygiene techniques and early detection methods.

New oral health technologies could provide patients and providers with valuable communication, education, and feedback leading to prevention of oral diseases. Monitoring one's oral health outside of the dental clinic is uncommon today, but $69 \%$ of adults track at least one health indicator so personal informatics technologies are used for many other health-related goals [4]. Researchers in HumanComputer Interaction (HCI) has proposed some ways that technology could support people in their oral health goals, including Lumio, DAYA, Playful Toothbrush, and Molarcropolis [5, 6, 7, 8]. However, there remains a large opportunity for technology to improve access to dental care and decrease the incidence of oral diseases.

In this research, we examine unmet information needs in oral care to identify opportunities for better monitoring at home and in connecting home care with clinical care. To do this, we first gained an understanding of patient and 
provider needs for an oral health monitoring technology through surveys and follow-up interviews. Then, we explored how clinical health workers currently bridge oral health care disparities in poor, rural areas with limited access to care by interviewing DHATs in Alaska, school nurses, and clinical health workers in low socioeconomic clinics, and dental providers. Together with a review of literature in dentistry, we identify the following opportunities for applying personal health informatics techniques to oral health:

- Facilitating communication between patient and provider

- Providing patient-centered care

- Visualizing oral health data to ensure early diagnosis

- Reducing oral health disparities

The HCI and Ubicomp research communities have already begun work on some of these needs pointing to promising future research directions. Our results provide guidance on what new dental monitoring tools must do to succeed.

\section{BACKGROUND AND MOTIVATION}

In this section, we examine causes for disparities in oral health in the United States, present how personal informatics technologies in other health conditions to promote collaboration between patient and provider, and discuss the opportunity for oral health monitoring technologies by reviewing related literature in HCI.

\section{Oral health disparities in the United States}

Among children aged 2-5, 23\% presented dental caries in their primary teeth, and $60 \%$ of adolescents aged 12-19 have dental caries in their permanent teeth [2]. The number of people who annually visit the dentist declines from $85 \%$ of children aged 2-17 to $64.0 \%$ of adults aged 18-64, and only $62.7 \%$ of adults over the age of 65 [9]. Along with a decline in dental visits, adults aged 40-64 were half as likely (29\%) to have all of their teeth compared to adults aged 20-39 [2, 10]. Age is not the only factor. NonHispanic white and Asian adults are on average 10\% more likely to have very good oral health compared to Hispanic or non-Hispanic black adults [11].

Dentists are expensive, highly trained, and are uncommon in rural areas. Among adults aged $18-64,42 \%$ did not visit the dentist due to cost or lack of insurance [2]. Adults without Medicaid are half as likely to visit the dentist and are five times as likely to have poor oral health as adults with private health insurance [2]. Many dental patients, however, do not need the skill of a dentist, but rather a hygienist or therapist [12]. Hygienists or therapists are less expensive than dentists to train and employ, and so there can be more of them per capita. For example, Dental Health Aide Therapists (DHATs) in Alaska are recruited from local communities and travel around Alaska from subregional clinics to spend weeks to months in remote villages without access to dental providers. DHATs are trained to perform preventative and restorative dental care and are supervised by a dentist working in a central city [3]. Rural dental providers often face problems with accessing patient's previous medical history and providing follow-up care [13]. In communities where there is a sub-regional clinic, year-round DHATs are able to do more maintenance and sealants. Also, DHATs provide annual screenings of every school child's teeth, scheduling them for follow-up exams and extractions as needed throughout the year.

\section{Collaboration with personal informatics}

Personal informatics technologies provide a space for users to set and achieve health-related goals while promoting healthy lifestyles changes and increasing knowledge about personal health. Personal informatics technologies seek to improve quality of life by understanding how technology can influence behavior and providing tools for education and health management [14]. Furthermore, personal informatics creates opportunities for contextual reflection, treatment adjustment, and communication with providers [15]. Contextual information allows the user to understand how their daily life, environment, and treatment impact their overall health [15]. For many health goals, personal informatics is most successful when continued for the longterm. However, keeping users motivated to track proves difficult when they feel uninterested or discouraged by their data [16]. Focusing on overall wellness in informatics technologies keeps users engaged by emphasizing that health tracking is not just about fixing a specific health condition, but rather a tool for maintaining a healthy life [17].

Personal informatics data allows patients to describe their everyday behavior, routine, and limitations when they review the data with providers. This information enables providers to understand patient experience better and to develop individualized diagnoses and treatment plans [18]. Providers can also use patient-tracked data as examples to educate patients on self-management skills $[18,19]$.

In oral health care, communication between patients and providers falls short surrounding patient concerns, education on proper hygiene techniques, and overall knowledge of personal oral health. The lack of successful communication is especially problematic for parents since they need to know how to properly educate their children. Along with communication, patient follow-up from the dentist rarely occurs in rural locations. One of the main reasons for difficulty in communication between patients and providers is the inability of patients to properly visualize their teeth [16]. Allowing patients to visualize their teeth and understand their oral health is a current area that needs more research [16].

\section{Oral monitoring research in $\mathrm{HCl}$}

Previous projects like LumiO, DAYA, Playful Toothbrush, and Molarcropolis have been designed to help individuals spend more attention on areas of their mouth that most need 
it and on helping parents monitor and encourage children's brushing $[5,6,7,8]$.

LumiO helps patients identify problematic areas in their mouth by using quantitative light fluorescence to determine how much plaque exists on each tooth [5]. As the user brushes their teeth and removes the plaque, the vibrations on the toothbrush decrease, giving the user immediate feedback on the quality of their brushing. Informed by this feedback, people using LumiO can use the feedback to allow their toothbrush to linger longer on teeth that have more plaque and thus hopefully improve their oral health. Three other systems have been designed to help parents monitor children's brushing and to motivate children to brush properly. DAYA is a mobile application for parents and a toothbrush that monitors how their children brush. The DAYA system was created on the basis that children do not understand the necessity of brushing their teeth, children are fearful of the dentist and cavities, and parents share the same concerns as their children but fail to give good instructions due to their lack of oral health knowledge [6]. Playful Toothbrush seeks to engage children and instill them with proper oral health habits by means of gamification [7]. The Playful Toothbrush aims to target children who are not interested in brushing their teeth by making the task interactive and fun. Also, the Playful Toothbrush educates children who do not brush their teeth properly. Along with Playful Toothbrush, Molarcropolis engages children and increases oral health awareness by using gamification in three ways: cause-and-effect simulations, suggestion, and attractiveness [8]. Cause-andeffect simulations quickly show users how their teeth would deteriorate with poor oral hygiene habits, suggestion provides an educational component, and the attractiveness of the system is tailored to the target audience.

\section{RESEARCH GOALS}

Existing oral health monitoring systems address important oral health needs beyond the dental clinic. They can help people monitor and self-regulate the frequency, intensity, and duration of their home care. New research prototypes can help people know when and where plaque is building up in their mouth and provide assistance for immediate oral health needs. Research focused on motivating and educating children to brush well has the potential to build important habits at a formative time.

In this research, we sought to step back from the goals and benefits of any one system to develop a better understanding of unmet information needs in dental care, focusing on the needs of adult patients, caregivers, and dental providers. We also sought to examine needs among underserved populations. While the prototype systems described above address important oral health concerns, they are designed for individual or individual family use. Pricey individual medical devices run the risk of "being out of reach of those who need them most", a charge that has been leveled in public discourse against wearables and other personal health technologies [20]. As a result, we sought to understand needs and opportunities for use of oral health monitoring that could help people across income levels, or especially people who are currently underserved by dental health care.

As we conducted our research, we used four principles of the quantified-self movement -- self-improvement, selfdiscovery, self-awareness, and self-knowledge -- to probe for opportunities, as they represent common information needs that can be supported by monitoring technology [21]. We also sought to identify individual patient goals beyond the oral health goals recommended by The Institute of Medicine Committee on Future of Dental Education. Considering these goals, such as whiter teeth, better breath, and reduced sensitivity, allowed our investigation to better take into account individual priorities and motivations.

\section{METHODS}

To explore opportunities for oral health monitoring technologies beyond the dental clinic, we gathered perspectives of personal users and medical providers. We first conducted surveys with 152 people who brush their teeth at least once a day and followed-up with eight participants in semi-structured interviews. To include a provider perspective, we conducted semi-structured interviews with twelve medical providers including six school nurses, two community health workers, one dentist, one dental assistant, and two Dental Health Aide Therapists who work in remote, rural populations.

\section{Surveys and Interviews about personal use}

To investigate opportunities for oral health monitoring at home, we first conducted a survey of 152 people ranging from 18 to over 75 years old that brush their teeth at least once a day. Table 1 shows survey participants grouped by age. We recruited participants through university mailing lists and posting the survey on Facebook and community groups. We included only participants over 18 years of age and who brushed their teeth at least once a day. We anticipated that people who do not already brush their teeth

\begin{tabular}{lcc}
\hline Participant ID & Count & Age \\
\hline S0-S49 & 50 & $18-24$ \\
\hline S50-S77 & 28 & $25-34$ \\
\hline S78-S102 & 25 & $35-44$ \\
\hline S103-S119 & 17 & $45-54$ \\
\hline S120-S136 & 17 & $55-64$ \\
\hline S137-146 & 10 & $65-74$ \\
\hline S147-S152 & 5 & $75+$ \\
\hline
\end{tabular}

Table 1: Survey participants 
once a day would not be likely to be interested in adding personal informatics tools to their oral health routine, though their motivations and needs merit future study. Each survey participant was entered into a raffle for one $\$ 100$ gift card and two $\$ 50$ gift cards. All participant data remains anonymous and participants could stop taking the survey or not answer a question.

The survey allowed us to ask a large number of people about their current oral health behaviors and attitudes, as well as where they wanted more support or information. We began by asking participants questions about their current oral care practices, their satisfaction with their oral health, and goals they might have with their oral health. Then, we asked about the patient's relationship with their dentists and how they communicate with their dentists. The following section of the survey focused on how participants track any other health-related goals and explored if oral health monitoring would be of interest. Finally, the survey asked participants with children at home about their experiences with helping their children with their oral care and maintaining good oral health.

Survey responses informed the design of the follow-up interviews and who we recruited. Quantitative data, like a ranking of oral health satisfaction, were analyzed by with descriptive statistics and visualizations. Qualitative data, like open response questions, were analyzed by coding for similar themes based on our research questions.

We selected follow-up interview participants to represent as many age groups as possible that we saw in survey results, and we randomly selected the participants within each group. Table 2 shows occupational information and teeth brushing frequency for interview participants. All follow-up interview participants signed a consent form stating that their answers would be completely confidential, they could choose not to answer any question, and they could withdraw from the study at any time. Each interview participant was compensated with a $\$ 25$ gift card. The goal of the thirty-minute semi-structured interview was to determine how personal informatics fits into their life, understand what information they might want from an oral health monitoring device, and get a sense of how oral health

\begin{tabular}{llll}
\hline Participant ID & Occupation & Teeth brushing freq & Age \\
\hline P1 & Student & Once a day & $18-24$ \\
\hline P2 & Business & Twice a day & $55-64$ \\
\hline P3 & Researcher & Once a day & $25-34$ \\
\hline P4 & Engineer & Once a day & $35-44$ \\
\hline P5 & Student & Twice a day & $18-24$ \\
\hline P6 & Homemaker & Once a day & $45-54$ \\
\hline P7 & Researcher & Once a day & $25-34$ \\
\hline P8 & Student & Twice a day & $18-24$ \\
\hline
\end{tabular}

Table 2: Interview participants monitoring might work best for them.

The interview was semi-structured so that each participant was asked the same questions, but as new needs or wants became apparent they could be explored in the interview. The interview began by asking participants about their oral health goals and knowledge about their oral health. Next, the interview asked about participant experiences with dentists, including receiving education and recommendations. Finally, the interview sought to discover how participants might use a wand that tracks the plaque load on their teeth. We asked participants more about how they track other health-related goals and asked them to describe how they would use a wand that helps them track oral health goals, easily see trends in the graphical data from quantitative measures of bacterial load, and monitor any therapeutic gains prescribed by their dentist. After the interviews, quotes were coded into eleven different themes based on apparent needs and wants of the participants.

\section{Interviews with oral health providers}

In our interviews with health providers, we included both current oral health providers and potential oral health providers. This reflects the potential of oral health technology to both improve existing oral health care and to create new opportunities for delivering oral health screening and treatment. We interviewed 12 health providers with a variety of occupations to learn about opportunities for medical providers using an oral health monitoring device for children and adults without access to regular dental care. Table 3 shows occupational details for interview participants. The semi-structured interviews allowed us to gather baseline data on roles and responsibilities for each community health worker while allowing each participant to go in a direction that felt important to them. We recruited school nurses and community health workers through online searches and public-school directories. The recruitment email stated that we were interested in learning about their experiences and needs as a school nurse and how an oral health-screening device might fit into their routines. All community health workers and school nurses signed a consent form stating that their answers would be completely confidential, they could choose not to answer any question, and they could withdraw from the study at any time. Each interview participant was compensated with a $\$ 25$ gift card.

The thirty-minute interview asked school nurses and

\begin{tabular}{lll}
\hline Participant ID & Count & Occupation \\
\hline M1-M6 & 6 & School nurse \\
\hline M7-M8 & 2 & Dental Health Aide Therapist \\
\hline M9-M10 & 2 & Community health worker \\
\hline M12 & 1 & Family dentist \\
\hline M13 & 1 & Hospital dentistry special assistant \\
\hline
\end{tabular}

Table 3: Provider participants 
community health workers about their roles and responsibilities, how often they have dental patients, experiences during dental visits, and how an oral health screening and tracking device might fit into their routine. Community health workers were asked about how often dental caries is present in their patients and what the treatment looks like. We also asked about how the school nurses and community health workers educate their patients and what recommendations they give. After the interviews were conducted, the interviews were coded with an open coding method. The codes were discussed and iterated as new themes became apparent.

\section{RESULTS}

Overall, participants described a desire for technology that could assist with oral health education, prevention, detection, and communication among family members and with oral health providers. Personal users were interested in monitoring their oral health to better understand their oral health, achieve personal goals, visualize their progress between dental visits for feedback, and properly adjust their home care routines. Among medical providers, there exists an opportunity for better screening and diagnostic tools to identify dental caries at early stages and to serve as a communication tool for patients and their caregivers. We find that oral health monitoring technologies have the opportunities to facilitate communication and coordination between patients and providers and to provide personalized education and feedback. Our results also show that monitoring oral health has the potential expanding access to oral health care in rural, underserved areas.

\section{Facilitating patient-provider communication}

People face many barriers in communicating with their oral health providers. Despite best efforts to educate patients, a dental assistant (M13) reported "we give these patients all this education, and yet they come back and look exactly the same." Medical providers also expressed difficulty with instructing patients about diet and abstaining from sugary sodas to reduce cavities. Despite best efforts from medical providers to communicate with their patients, the information was often not received. A family dentist (M12) reflected on communicating with their patients: "prevention is the key. People don't go [to the dentist]. They don't like us. They don't like dental offices. They don't like dentists." When community health workers had time to do education, they primarily instructed people on how to brush and floss properly, but many patients learned incorrect brushing techniques from their parents. Therefore, community health workers often had to be creative about how they communicate with their patients. A DHAT (M7) educated their patients using a unique method: "Sometimes I use metaphors, like 'where else do you have germs? On our hands. What do you do to take the germs off your hands?' They tell me they wash their hands. I ask them, 'what happens if you don't wash your hands?' They usually say they get sick, and then I use that as a metaphor."
From a patient perspective, P5 found that their dentist "just told me I didn't have much problems and they gave free samples offloss and that's it. They didn't really tell me what I should do for my teeth though." Finding the right questions to ask their dentist is also hard for personal users, so many resorted to asking general questions like "is everything ok?" (P4). While providers were able to gather information from a visual examination of the mouth, P4 found it frustrating that "they don't really ask if I' $m$ flossing or anything." When reflecting on their communication with their dentist, P8 recounted that their "dentist talks kind of fast, but I think the use of images and you know like 3D modeling or things like that, that would be really useful, because it's hard for me to, when she describes teeth, and tries to kind of draw it out, it's a very like 1D, just linear tooth model. It's really difficult for me to visualize which tooth she's talking about, so I think just some kind of visualization product would be really cool when talking about a patient's teeth."

\section{Providing personalized education and feedback}

Personal users and community health workers expressed concerns about lack of oral health knowledge. Preventing tooth decay is the number one oral health goal for 125 out of 152 survey respondents, yet 86 survey respondents expressed that proper home care, mainly brushing techniques and frequency of flossing, remains their main confusion surrounding oral health. 76 out of 152 survey respondents indicated that they would like to monitor the amount of plaque buildup on their teeth. Specifically, S87 responded that they would "like to know which teeth, if any, are at risk for cavities, and how bad the damage is." Many personal users rely on self-discovery for oral health. For example, P4 recounted that they simply "know the areas of my mouth that I need to give extra attention to."

Retaining oral health knowledge after appointments was also difficult. P6 stated: "right after I finish the dentist, I know quite a bit. But before I go to the dentist, not as much." Personal users wanted more specific feedback from their dentist and were not able to tell if the recommendations are working. P1 described that they "want much more practical information about the mechanics of flossing. Does it appear that I'm doing something right, something wrong? I don't know. Just the more practical advice that maybe you've always just assumed you know how to do something the right way and maybe you don't." Personal users were interested in gaining oral health knowledge for increasing overall health and debunking confusion around dental visits. P3, who struggled with extreme dental fear, thought "that it would help allay some of my dental fears if I actually knew what was going on, like if I walked into the next dental appointment and wasn't completely freaked out by the unknown." Personal users were also interested in learning about their oral health for prevention, P2 stated "had I jumped on board of preventing enamel damage earlier, 
rather than trying to just put a Band-Aid fix now, I think that that would have been really good."

Community health workers focus on providing "basic oral hygiene" (M5) due to an almost complete lack of oral health knowledge in most patients. In particular, parents often do not have sufficient oral health knowledge to pass on to their children. One dentist (M10) noted that oral health knowledge is "lacking in $80 \%$. Then, so I give advice. Just make sure your child brushes. If you let your child brush his or her teeth, then, do it right. They do it the best they can, but they don't know what's the best." When reflecting on the ability to get children involved in proper oral hygiene, one school nurse (M6) stated that "this past school year, I had a lot of trouble getting the middle school boys to brush. It was very difficult working with them this year. So I gotta try to figure out a better way of getting them intrigued with taking care of their oral health."

\section{Monitoring oral health for early diagnostics}

Community health workers would benefit from an oral health monitoring tool as a better diagnostic tool of dental caries at an earlier stage. It is difficult for providers to tell if a tooth is just discolored or if there is decay based on a visual assessment. For extreme cases of caries, such as those between the back teeth, it is also difficult to tell how deep it is until the restorative treatment takes place.

An oral health monitoring system used as a diagnostic tool would allow community health workers in rural areas, like traveling DHATs, an opportunity to easily see trends in patient bacterial load on each tooth and make early and accurate diagnoses. A DHAT (M8) expressed a general need and ability to incorporate a caries detection tool in their day-to-day work due to the volume of dental caries and poor oral hygiene. In regard to providing early detection of caries by using an oral health monitoring device to detect plaque loads, one community health worker (M10) said "if there was a fairly accurate, I would say I would need to be at least $80 \%$ accurate or something that it is a cavity and not a stain, then we could treat cavities when they're smaller. It's always easier to fix a tooth when the cavity's small than when it's bigger. So essentially, as soon as you start getting a filling, like the rest of your life, that tooth is probably going to need another thing later on because fillings only last, I don't know, ten, fifteen years. If you're young, eventually it's going to have to be replaced and every time it gets replaced, it gets bigger."

\section{Expanding access to oral health care}

We found potential for expanding access to oral health care with oral health monitoring by creating a low-cost, portable, time-efficient device. Some DHATs, like M7, have programs to work in schools and screen every student in their town, but in villages where the DHATs must travel to have less care, so their time is focused on providing extractions rather than education and sealants. The ability to provide education is crucial, because most oral diseases, including dental caries, are preventable with proper education on basic hygiene practices. Both DHATs (M7 \& M8) expressed interest in a tool that monitors and educates patients about their oral health. Adopting a new monitoring device for rural dental providers and community health workers can be difficult due to poor communication infrastructure, time and cost of technical training, and installation and maintenance costs. However, M8 talked about how "if it was portable, or it could roll to the school, 'cause I do my school screenings in the school, I'd use it in the school and I'd use it in the clinic. Then that would be good to help monitor if the decay is getting larger or it is porous. But I think that's a really good idea and I think it would really help me." M7 also stressed portability and the need for an oral health monitoring device to be small "because you have to take it on a plane with you and there's a lot of gear we have to take" when travelling to remote Alaskan villages.

Since education and prevention are paramount for good oral health, we find that focusing on children who are still developing oral health habits to be the population in most need. However, all 6 school nurses interviewed work at multiple schools, making it difficult to implement consistent education and prevention programs. Requiring annual dental screenings is done by the state, and few states have chosen to require such screening. Without state requirements, busy school nurses lack time, funding, and resources to incorporate oral health monitoring for their students. One school nurse (M1) expressed that they have "too much work, not enough time. You can't do everything, because it's not possible. So, with my four things I have 1400 kids for me. I just have to start letting things go." 4 of 6 school nurses expressed being overwhelmed by mandated responsibilities, and they have little time to do other things. Schools have limited funding, and expensive equipment makes it difficult to change or add new tools into school nurses' routines. For example, near vision screenings have been added to the list of Washington State mandated screenings. However, the tool needed to for the vision screening is too expensive for every school to buy. With funding in mind, a school nurse stated that they are "It's always grant writing and you know just trying to scrape together money because our health services don't really have a budget aside from just to pay staff." 5 school nurses explained how their schools rely on outside community health agencies to ensure every student has at least one annual dental screening. However, school nurse (M3) expressed disappointed that another parental consent was required for the dental screenings by community health agencies, and so not all students were actually able to get a dental screening. School nurse M2 spoke about their sadness and frustration regarding the lack of young parent oral health education and the parents' inability to stress good oral health with their young kids: "Too many children have been seen in elementary schools with fillings in their 
baby teeth, which sets them up to have more overall health problems in the future."

Oral health monitoring technologies used outside of the dental clinic could reduce oral health disparities by increasing access to dental care. While lack of time and funding presents problems for school nurses, oral health monitoring technologies can aid community health workers who provide dental screenings to schools or DHATs in Alaska.

\section{DISCUSSION}

We found that personal users and medical providers present specific wants and needs regarding oral health education, communication, prevention, and diagnosis of oral diseases. We discuss how monitoring of oral health by personal users and medical professionals can reduce oral health disparities by providing access to basic oral health care, streamlining dental visits, and creating awareness of proper oral hygiene practices in families and children.

\section{Bridging dental expertise and personal practice}

Currently, dentists, community health workers, or other trained experts are necessary for people to assess their oral health and monitor whether many problems, such as carries, are worsening or improving. As a result, patients often follow their home care routine with little feedback about whether they are doing it correctly or whether it is achieving the desired results. They may not discover problems until more serious measures, such as a filling, are needed. From an oral health professional's perspective, when patients arrive at the clinic appointment, health professionals can only see the results (e.g., whether patients have cavities) but not what patients have tried (e.g., if and how well they use floss).

When describing what to do at home, oral health providers often rely on verbal descriptions and demonstrations on themselves or the patient. This in-person demonstration enables patients to ask questions, but the details may be hard to remember. Once home, the patient may forget which tooth exactly they were supposed to brush better, which flossing technique their hygienist demonstrated, or the advice entirely.

Personal health technologies can help bridge the gaps between clinical encounters, home care, and other everyday behaviors (e.g., in diabetes [19], IBS, weight management [22] and Parkinson's disease [23]). Summaries and data about home care and summaries of recommendations made during a clinical encounter can help make work that both patients and their providers do more effective.

\section{Making better use of patient time and clinical resources}

Bridging home care and the clinic also has the potential to help people make better use of their time and dental resources. Home monitoring or monitoring available in a community setting (e.g., a kiosk in a pharmacy or community center) could help people determine whether they need to see a dental provider. If patients become aware of developing problems in time and can adjust their care routine or apply a therapy, it may prevent a visit as well. This is particularly important for people in rural areas, where access to clinical care may cost significant time and inconvenience. Similar techniques, e.g., blood pressure kiosks in clinic waiting rooms or pharmacies [22], have already shown promise for making patients more engaged with their health, helping patients access health resources at convenient locations and times, and allowing health professionals to spend time on other forms of care rather than routine measurements.

\section{Collective Oral health}

Our results also support HCI's focus on oral health as a family concern [24]. This previous research focuses on encouraging children to adopt healthy brushing habits. Inspired by recent research in family tracking [24] however, we believe this may be just one of a larger set of opportunities for supporting oral health within families.

While parents can monitor children's oral care, shared awareness of oral care could also create the sense of this being a family activity. In situations when one family member has a particular oral health concern, technologies might be designed to help the family support them [24]. More research is needed, though, to understand opportunities here, as there is also the potential for this to become a nag, a surveillance tool, or another annoyance for busy families to deal with.

Tools that support monitoring in the home might also create opportunities for parents and children to better educate themselves about the efficacy of different oral care routines. If sensitive enough, they might even support selfexperiments [25]: which flossing technique is better for me? How much better is my electric toothbrush than my manual brush? If I remove carbonated drinks from my diet, do I see an improvement?

\section{Limitations}

In our recruitment, we emphasized inclusion of parents and oral health providers who could offer perspectives on working in rural areas. As a result, our participants underrepresented the elderly and possibly other groups. Additional opportunities may exist for oral health challenges unique to or more prevalent in these groups, and future research should examine their needs and associated opportunities in depth.

Researching DHATs in Alaska provided insight into oral health monitoring technologies might fit into a clinical setting, but there is a large scope of clinicians and community health workers and we were only able to interview in a few domains. Finally, our survey methodology produces a limitation in that it is common that what people say they do and what they actually do tend to differ, so it would be difficult to draw specific design recommendations from this data. However, we believe 
results are able to specify clear needs and opportunities for future technologies to address.

\section{CONCLUSION}

We contribute to the field of personal informatics technologies by describing opportunities and limitations for oral health monitoring technologies at home and beyond the dental clinic.

We surveyed 152 people that brush their teeth at least once a day and followed up with 8 semi-structured interviews. We also interviewed 12 medical providers with a range of professions in a semi-structured format.

We learned about the wants and needs of personal users by studying how they keep track of their oral health, understanding successes and limitations in currently used personal informatics devices, and learning about how oral health monitoring might fit into their routines. We studied the wants and needs of medical providers for oral health monitoring technologies by understanding their roles and responsibilities, discovering how they interact with dental patients, and gaining insights into how oral health monitoring would work best for their position.

We found four distinct opportunities for oral health monitoring technologies beyond the dental clinic: (1) Facilitating communication between patient and provider (2) Providing personalized education and feedback (3) Monitoring oral health for early diagnostics (4) Expanding access to oral health care. We further discussed opportunities for oral health monitoring technologies that can bridge dental expertise and personal practice, make better use of patient time and clinical resources, and promote oral health as a collective effort.

\section{ACKNOWLEDGMENTS}

This research was funded by National Science Foundation awards \# IIP-1631146 and IIS-1553167. We thank Morgan Duffy for preliminary work that laid important groundwork for this research.

\section{REFERENCES}

1. BA Dye, S Tan, V Smith, BG Lewis, LK Barker, G Thornton-Evans, PI Eke, ED Beltran-Aguilar, AM Horowitz, CH Li. 2007. Trends in oral health status, United States, 1988-1994 and 1999-2004. Vital Health Stat 11; (248):1-92.

2. B Bloom, CM Simile, PF Adams, RA Cohen. 2012. Oral health status and access to oral health care for U.S. adults aged 18-64: National Health Interview Survey, 2008. National Center for Health Statistics. Vital Health Stat 10(253).

3. Donald Chi, Dane Lenaker, Lloyd Mancl, Matthew Dunbar, Machael Baab. 2017. Dental Utilization for Communities Served by Dental Therapists in Alaska's Yukon Kuskokwim Delta: Findings from an
Observational Quantitative Study. University of Washington Press Release.

4. Susanna Fox, Maeve Duggan. 2013. Tracking for Health. Pew Research Center.

http://www.pewinternet.org/2013/01/28/tracking-forhealth/

5. Takuma Yoshitani, Masa Ogata, Koji Yatani. 2016. LumiO: A Plaque Aware Toothbrush. Proceedings of the 2016 ACM International Joint Conference on Pervasive and Ubiquitous Computing: 605-615. https://doi.org/10.1145/2971648.2971704

6. Kejia Shao, Jiye Huang, Huaying Song, Runze Li, Jinxi Wu. 2014. DAYA: a system for monitoring and enhancing children's oral hygiene. CHI '14 Extended Abstracts on Human Factors in Computing Systems: 251-256. https://doi.org/10.1145/2559206.2580927.

7. Yu-Chen Chang, Jin-Ling Lo, Chai-Hu Huang, Nan-Yi Hsu, Hai-Hua Chu, Hsin-Yen Wang, Pei-Yu Chi, YaLin Hsieh. 2008. Playful Toothbrush: UbiComp Technology for Teaching Tooth Brushing to Kindergarten Children. Proceedings of the SIGCHI Conference on Human Factors in Computing Systems: 363-372. https://doi.org/10.1145/1357054.1357115

8. Carmen Soler. Alejandra Zacarias, Andres Lucero. Molarcropolis: a mobile persuasive game to raise oral health and dental hygiene awareness. 2009. Proceedings of the International Conference Advances in Computer Entertainment Technology.: 388-391. https://doi.org/10.1145/1690388.1690468

9. BA Dye, X Li, G Thornton-Evans. 2012. Oral health disparities as determined by selected Healthy People 2020 oral health objectives for the United States, 20092010. NCHS data brief, no 104. Hyattsville, MD: National Center for Health Statistics.

10. BA Dye, G Thornton-Evans, X Li, TJ Iafolla. 2015. Dental caries and tooth loss in adults in the United States, 2011-2012. NCHS data brief, no 197. Hyattsville, MD: National Center for Health Statistics.

11. BA Dye, G Thornton-Evans, X Li, TJ Iafolla TJ. 2015. Dental caries and sealant prevalence in children and adolescents in the United States, 2011-2012. NCHS data brief, no 191. Hyattsville, MD: National Center for Health Statistics.

12. N. P. Monaghan, M. Z. Morgan. 2015. What proportion of dental care in care homes could be met by direct access to dental therapists or dental hygienists? British Dental Journal: 531-534. https://doi.org/10.1038/sj.bdj.2015.919.

13. Hossein Pourezza, Sergio Camorlinga, Carson K.S. Leung, Bruce Martin. 2010. An information and communication technology system to support rural 
healthcare delivery. Proceedings of the $1^{\text {st }}$ ACM International Health Informatics Symposium: 440-444. https://doi.org/10.1145/1882992.1883059

14. Francisco Nunes. 2015. Designing Self-care Technologies for Everyday Life: A Practice Approach. Proceedings of the $33^{\text {rd }}$ Annual ACM Conference Extended Abstracts on Human Factors in Computing Systems: 215-218. https://doi.org/10.1145/2702613.2702624

15. Francisco Nunes, Nervo Verdezoto, Geraldine Fitzpatrick, Morten Kyng, Erik Gronvall, Cristiano Storni. 2015. Self-care Technologies in HCI: Trends, Tensions, and Opportunities. Proceedings of the Transactions on Computer-Human Interaction. https://doi.org/10.1145/2803173.

16. Amid Ayobi, Paul Marshall, Anna L. Cox. 2016. Reflections on 5 Years of Personal Informatics: Rising Concerns and Emerging Directions. Proceedings of the 2016 CHI Conference Extended Abstracts on Human Factors in Computing Systems: 2774-2781. https://doi.org/10.1145/2851581.2892406

17. Rebecca E, Grinter, Katie A. Siek, Andrea Grimes. 2010. Wellness informatics: towards a definition and grand challenges. CHI '10 Extended Abstracts on Human Factors in Computing Systems: 4505-4508. https://doi.org/10.1145/1753846.1754185.

18. Chia-Fang Chung, Kristin Dew, Allison Cole, Jasmine Zia, James Fogarty, Julie Kientz, Sean Munson. 2016. Boundary Negotiating Artifacts in Personal Informatics: Patient-Provider Collaboration with Patient-Generated Data. Proceedings of the ACM Computer Supported Cooperative Work Conference.

19. Lena Mamykina, Elizabeth Mynatt, Patricia Davidson, Daniel Greenblatt. 2008. MAHI: Investigation of Social Scaffolding for Reflective Thinking in Diabetes Management Proceedings of the SIGCHI Conference on Human Factors in Computing Systems: 477-486

20. J Elias. 2015. Why Wearables are Out of Reach for People Who Need Them Most. Forbes Magazine. https://www.forbes.com/sites/jenniferelias/2015/10/27/ the-leftovers-part-i-the-cost-ofactivity/\#791cd70d63c9.

21. Gary Wolf. 2010. The Quantified Self. Ted@ Cannes. https://www.ted.com/talks/gary_wolf_the_quantified_s elf

22. Chia-Fang Chung, Sean A. Munson, Matthew Thompson, Laura-Mae Baldwin, Jeffrey Kaplan, Randall Cline, Beverly B. Green. 2016. Implementation of a New Kiosk Technology for Blood Pressure Management in a Family Medicine Clinic: from the WWAMI Region Practice and Research
Network. The Journal of the American Board of Family Medicine 29.5: 620-629.

23. Helena Mentis, Anita Kolmlodi, Katrina Schrader, Michael Phipps, Ann Gruber-Baldini, Karen Yarbrough, Lisa Shulman. 2017. Crafting a View of Self-Tracking Data in the Clinical Visit. Proceedings of the 2017 CHI Conference on Human Factors in Computing Systems: 5800-5812. https://doi.org/10.1145/3025453.3025589

24. Laura Pina, Sang-wha Sien, Teresa Ward, Jason Yip, Sean Munson, James Fogarty, Julie Kientz. 2017. From Personal Informatics to Family Informatics: Understanding Family Practices around Health Monitoring. Proceedings of the 2017 ACM Conference on Computer Supported Cooperative Work and Social Computing: 2300-2315. https://doi.org/10.1145/2998181.2998362

25. Ravi Karkar, Jasmine Zia, Roger Vilardaga, Sonali R Mishra, James Fogarty, Sean A Munson, Julie A Kientz; 2016. A framework for self-experimentation in personalized health, Journal of the American Medical Informatics Association, 23(3): 440-448. https://doi.org/10.1093/jamia/ocv150 